\title{
A STUDY OF ULTRAVIOLET SPECTRA OF DELTA SAGITTAE
}

\author{
A. AKALIN and S. ENGIN \\ University of Ankara \\ Dept. of Astronomy and Space Sciences \\ 06100 Tandogan, Ankara \\ Turkiye
}

\begin{abstract}
High resolution spectrum of the VV Cephei type star $\delta$ Sge (M2 II + A0 V) observed with the IUE satellite between 1979-1985 have been analysed. The radial velocity curves of non blended absorption features have been formed. Observed radial velocities are compared with the theoretical radial velocity curve due to orbital motion. We found that observed radial velocity variation is the resultant of radial velocity of hotter component and wind velocity around this star. The wind is perturbed by hotter component. Matching the UV continuum fluxes obtained from IUE high resolution spectra to fluxes of Kurucz model atmospheres yields $\mathrm{Te}=11000 \pm 500^{\circ} \mathrm{K}$. Thus the spectral type of hot component was found as $\mathrm{A} 0$.
\end{abstract}

\section{Introduction}

Delta Sagittae - classified as M2 II+B by Batten and Fisher (1981) and M2 II+A0 V by Batten (1986) is a spectroscopic binary of VV Cephei type with an orbital period of 3720 days. B type component is eclipsed by the atmosphere of the $\mathrm{M}$ giant.The system must have an orbital inclination greater than $70^{\circ}$, since atmospheric eclipses have been observed. The last periastron passage occurred in the second week of April 1990.

Distance to the system was determined 300 pc by Reimers and Kudritzki (1981), 224 pc by Batten and Fisher (1981) and $170 \pm 30 \mathrm{pc}$ by Reimers and Schroder (1983).

\section{Observations}

We have collected twelve IUE images of $\delta$ Sge from to IUE-VILSPA data bank, The spectra cover the period between May 26, 1979 and May 14, 1985. All spectra are high resolution and large aperture spectra. Table. 1 shows the list of the images. The phases have been computed by the ephemeris minimum $=J D 2444271+3720^{d}$. E (Reimers and Schroder, 1985).

\section{Line Identification}

In all spectra, more than 700 lines have been identified between 1200 and $3000 \AA$ which shows numerous sharp absorption lines, and P Cygni and inverse P Cygni features on the B star's continuum. 
TABLE 1 . The high resolution and large aperture images

\begin{tabular}{lcccr}
\hline Image & Camera & Date & Phase & Exp.(s) \\
\hline L4606 & LWR & 26.05 .1979 & 0.93 & 600 \\
S6833 & SWP & 11.10 .1979 & 0.97 & 2640 \\
L5810 & LWR & 11.10 .1979 & 0.97 & 1740 \\
S9649 & SWP & 30.07 .1980 & 0.05 & 2700 \\
L8396 & LWR & 30.07 .1980 & 0.05 & 1800 \\
S16664 & SWP & 30.03 .1982 & 0.21 & 3900 \\
L12903 & LWR & 30.03 .1982 & 0.21 & 2400 \\
S21070 & SWP & 16.09 .1983 & 0.36 & 2700 \\
L16807 & LWR & 16.09 .1983 & 0.36 & 1200 \\
L4095 & LWP & 27.08 .1984 & 0.45 & 1980 \\
S25942 & SWP & 14.05 .1985 & 0.52 & 1290 \\
L5982 & LWP & 14.05 .1985 & 0.52 & 1500 \\
\hline
\end{tabular}

Fe II lines are dominated in the UV spectrum of $\delta$ Sge. In addition singly ionized metal lines as Ni II, Cr II, Mn II are seen. This lines varies in phase and such variations are different for different lines. Furthermore, the UV spectra includes high ionization lines such as C IV, Al III and Si IV. This broad line profiles also vary in phase and the lines are deep broad absorption features during to eclipse however they are less broad and sometimes have emission component outside eclipse. The high ionization lines do not arise from the hot star but $\delta$ Sagittae system has an high temperature region which forms high ionization lines. According to Reimers and Kudritzki (1981), the high temperature could be produced by shoch heating when the potential energy of infalling matter is transformed into kinetic energy.

\section{Radial Velocities}

We have measured the radial velocity of non blended and only absorption features (Table 2). Theoritical radial velocity curves of $\delta$ Sge's components obtained by using Batten's (1986) new orbital elements were compared with the observed radial velocities (Figure 1).

TABLE 2. Mean velocity values as $\mathrm{km} \cdot \mathrm{s}^{-1}$

\begin{tabular}{ccccccccc}
\hline & \multicolumn{1}{c}{ Line } & \multicolumn{7}{c}{ PHASES } \\
Element & Mult. & Number & 0.97 & 0.05 & 0.21 & 0.36 & 0.52 \\
\hline \multirow{2}{*}{ Fe II } & 9 & 2 & 20.4 & 10.5 & -7.4 & -41.9 & -18.3 \\
Fe II & 38 & 3 & 22.1 & 8.8 & -22.7 & -42.9 & -30.4 \\
Fe II & $40,41,42$ & 18 & 27.8 & 7.6 & -21.6 & -34.9 & -29.3 \\
& $43,44,45,47$ & & & & & & \\
Ni II & $4,5,8$ & 3 & 20.9 & 7.1 & -21.0 & -36.6 & -25.4 \\
\hline
\end{tabular}


Reimers and Schroder (1983) was found that the extended envelope of M giant expands with 25 to 30 $\mathrm{km} . \mathrm{s}^{-1}$. The observed radial velocity variation looks resultant of radial velocity of hot component and wind velocity around this star. The wind is perturbed by hot component. Reimers and Schroder (1989) studied Ca II lines in optical region. They found the expansion velocity of envelope of $\mathbf{M}$ giant component.

\section{B Star}

The ultraviolet spectra of atmospheric eclipsing binaries are dominated by the radiation of hot component. We derived UV continuum and matching the continuum fluxes to fluxes of Kurucz model atmospheres yields $\mathrm{Te}=11000^{\circ} \mathrm{K} \pm 500^{\circ} \mathrm{K}$. The observed fluxes have been corrected with an average interstellar reddening of $E(B-V)=0.03$. The correction is too small in the invisibility of the interstellar absorption feature at $2200 \AA$ A. Figure 2 shows UV energy distribution curve of $\delta$ Sge, and theoreticalfit by Kurucz model atmospheres. Thus, we founded spectral type of hot component of $\delta$ Sagittae as A0.

\section{Results}

^ The UV spectra includes high ionization lines such as C IV, Al III and Si IV which vary in phase.

^ The observed radial velocity variation looks resultant of radial velocity of hot component and wind velocity around this star. The wind is perturbed by hot component.

^ Matching the UV continuum fluxes to fluxes of Kurucz model atmospheres yields $\mathrm{T}_{\mathrm{e}}=11000^{\circ} \mathrm{K} \pm 500^{\circ} \mathrm{K}$.

^ The UV energy distribution curve of $\delta$ Sagittae was fitted by Kurucz model atmospheres and founded spectral type of hot component of $\delta$ Sagittae as A0.

\section{References}

Batten, A. and Fisher, W. A. (1981) 'Spectroscopic evidence for an atmospheric eclipse of $\delta$ Sagittae', Publications of the Astronomical Society of the Pacific, 93, 769-771.

Batten, A. (1986) 'Interm discussion of the orbit of $\delta$ Sagittae', Highlight of Astronomy, J. P. Swings (ed.), Reidel Publ., Dordrecht, 207-209.

Reimers, D. and Kudritzki, R. P. (1981) 'Observational evidence for an accretion disk around the B star companion of $\delta$ Sge (M2 II)', Proceedings of Second European IUE Conference, Tubingen, Germany, 26-28 March 1980 (ESA SP-157), 229-232.

Reimers, D. and Schroder, K. P. (1983) 'A study of UV spectra of $\zeta$ Aur/VV Cep stars' , Astronomy and Astrophysics, 124, 241-246.

Reimers, D. and Schroder, K. P. (1989) 'Observations of modulation and phase displacement of the stellar wind in six red giant spectroscopic binaries', Astronomy and Astrophysics, 214, 261-266. 